Molecular Physics, 1992, Vol. 77, No. 6, 1021-1031

\title{
Effect of a density-dependent potential on the phase behaviour of fluids
}

\author{
By B. SMIT \\ Koninklijke/Shell-Laboratorium, Amsterdam (Shell Research B.V.), PO Box 3003, \\ 1003 AA Amsterdam, The Netherlands \\ and Department of Chemistry, University of California, Berkeley, \\ California 94720, USA

\section{T. HAUSCHILD and J. M. PRAUSNITZ} \\ Chemical Engineering Department and Chemical Science Division, \\ Lawrence Berkeley Laboratory, University of California, Berkeley, \\ California 94720, USA
}

(Received 14 May 1992; accepted 31 May 1992)

This work studies the effect of density-dependent potentials on the phase behaviour of fluids. For a particular class of density-dependent potentials, the equation of state has a simple relation to the equation of state of a reference fluid with a density-independent potential. This relation is illustrated by computer simulations of a fluid with density-dependent Lennard-Jones potential. Density-dependent potentials can be used in the Gibbs ensemble; this work presents the appropriate expressions for the pressure and chemical potential.

\section{Introduction}

In most computer simulations of liquids, interactions between molecules are modelled using two-body potentials [1]. These potential are obtained by fitting to experimental data. Real fluids, however, interact with three and higher-body interactions as well; therefore, these two-body potentials represent, in fact, effective interactions. To calculate accurate phase equilibria, it may be necessary to use a different two-body potential for the liquid phase and the gas phase. Simulations using two, three and higher-body interactions are at present too expensive for routine calculations [2,3]. A practical method to take three and higher-body interactions into account is provided by using pair potentials that depend on density. We investigate here a particular class of density-dependent potentials, those that are written as

$$
U_{\mathrm{d}}=f(\rho) U_{0},
$$

where $U_{\mathrm{d}}$ is the density-dependent potential, $f(\rho)$ is an arbitrary function of the density such that $f(\rho)$ becomes unity as density $\rho$ goes to zero. $U_{0}$ is a densityindependent potential which governs low-density properties such as the second virial coefficient. We show that the equation of state of a fluid that can be described with a density-dependent potential $U_{\mathrm{d}}$ is related to the equation of state of a fluid whose potential is $U_{0}$.

In Section 2, we investigate the influence of the density-dependent potential on the equation of state. Section 3 discusses the consequences of a density-dependent 
potential for computer simulations in the $N, V, T$ and Gibbs ensemble. As a particular example, we consider the Lennard-Jones fluid in Section 4.

\section{Equation of state}

Consider a fluid that is modeled with a density-dependent potential $U_{\mathrm{d}}$. We assume that the density dependence can be described using

$$
U_{\mathrm{d}}=f(\rho) U_{0},
$$

where $f(\rho)$ is an arbitrary function of the density $\rho=N / V, N$ is the number of particles and $V$ is the volume. $U_{0}$ is the density-independent potential of the reference fluid. The partition function of the fluid with density-dependent potential is given by

$$
Z(\beta, N, V)=\frac{1}{\Lambda^{3 N} N !} \int \mathrm{d} \Gamma^{N} \exp \left(-\beta U_{\mathrm{d}}\right)
$$

where $\beta=1 / k_{\mathrm{B}} T, A=\left(2 \pi \hbar^{2} \beta / m\right)^{1 / 2}$ is the thermal de Broglie wavelength, and $\Gamma^{N}=r_{1} \cdots r_{N}$ denotes the $3 N$ coordinates of the particles. The partition function of the reference fluid is given by

$$
Z_{0}(\beta, N, V)=\frac{1}{\Lambda^{3 N} N !} \int \mathrm{d} \Gamma^{N} \exp \left(-\beta U_{0}\right) .
$$

Substitution of equation (2) into equation (3) relates the partition function of the fluid with a density-dependent potential to that of the reference fluid

$$
Z(\beta, B, V)=\frac{1}{\Lambda^{3 N} N !} \int \mathrm{d} \Gamma^{N} \exp \left[-\beta f(\rho) U_{0}\right]=\left(\frac{\beta^{\prime}}{\beta}\right)^{(3 / 2) N} Z_{0}\left(\beta^{\prime}, N, V\right),
$$

with

$$
\beta^{\prime}=\beta f(\rho) .
$$

From these expressions, it follows (see Appendix A for details) that the equation of state $(P)$ of the fluid with the density-dependent potential can be expressed in terms of the equation of state $\left(P_{0}\right)$ and energy per particle $\left(u_{0}\right)$ of the reference fluid. This equation of state is given by

$$
P(\beta, \rho)=f(\rho) P_{0}\left(\beta^{\prime}, \rho\right)+\left(u_{0}\left(\beta^{\prime}, \rho\right)-\frac{3}{2 \beta f(\rho)}\right) \frac{\mathrm{d} f(\rho)}{\mathrm{d} \rho} \rho^{2} .
$$

If the equation of state of the reference fluid is known, we can obtain the equation of state for the fluid with the density dependent potential using equation (7).

\section{Computer simulations}

We now consider a simulation in the Gibbs ensemble $[4,5]$. The partition function of the Gibbs ensemble, using a density-dependent potential, is given by $[6,7]$

$$
\begin{aligned}
\bar{Z}(\beta, N, V)= & \frac{1}{V \Lambda^{3 N} N !} \sum_{n_{1}=0}^{N}\left(\begin{array}{l}
N \\
n_{1}
\end{array}\right) \int_{0}^{V} \mathrm{~d} V_{1} \int \mathrm{d} \Gamma_{1}^{n_{1}} \exp \left[-\beta f\left(\frac{n_{1}}{V_{1}}\right) U_{0}\right] \\
& \times \int \mathrm{d} \Gamma_{2}^{N-n_{1}} \exp \left[-\beta f\left(\frac{N-n_{1}}{V-V_{1}}\right) U_{0}\right]
\end{aligned}
$$

where $n_{1}$ denotes the number of particles in box 1 and $V_{1}$ the volume of box 1. To 
distinguish the partition function of the Gibbs ensemble from that for the canonical ensemble, we denote the former by $\bar{Z}$. This partition function can be rewritten in terms of a product of two partition functions of the canonical ensemble

$$
\bar{Z}(\beta, N, V)=\frac{1}{V} \sum_{n_{1}=0}^{N} \int_{0}^{V} \mathrm{~d} V_{1} Z\left(\beta, n_{1}, V_{1}\right) Z\left(\beta, N-n_{1}, V-V_{1}\right)
$$

where

$$
Z\left(\beta, n_{1}, V_{1}\right)=\frac{1}{\Lambda^{3 n_{1}} n_{1} !} \int \mathrm{d} \Gamma_{1}^{n_{1}} \exp \left[-\beta f\left(\frac{n_{1}}{V_{1}}\right) U_{0}\right]
$$

Using equation (9), we can define the Helmholtz energy of the Gibbs ensemble

$$
\bar{A}=\frac{1}{\beta} \ln \bar{Z},
$$

or in terms of the Helmholtz energies in the canonical ensemble

$$
\bar{A}(\beta, N, V)=\sum_{n_{1}=0}^{N} \int_{0}^{V} \mathrm{~d} V_{1}\left[A\left(\beta, n_{1}, V_{1}\right)+A\left(\beta, N-n_{1}, V-V_{1}\right)\right] .
$$

In [6] it is shown that for a sufficiently large system, the dominant contributions to the Helmholtz energy $\bar{A}$ are from those values of $n_{1}$ and $V_{1}$ where $A\left(\beta, n_{1}, V\right)+A\left(\beta, N-n_{1}, V-V_{1}\right)$ has its minimum. As a result, the Gibbsensemble simulations will find the $n_{1}$ and $V_{1}$ that satisfy

$$
\left(\frac{\partial\left[A\left(\beta, n_{1}, V_{1}\right)+A\left(\beta, N-n_{1}, V-V_{1}\right)\right]}{\partial V_{1}}\right)_{\beta, n_{1}}=0
$$

and

$$
\left(\frac{\partial A\left[\left(\beta, n_{1}, V_{1}\right)+A\left(\beta, N-n_{1}, V-V_{1}\right)\right]}{\partial n_{1}}\right)_{\beta, V_{1}}=0 .
$$

The derivatives of the Helmholtz energy with respect to the volume or number of particles define the pressure and chemical potential, respectively. Thus, these two expression demonstrate that the Gibbs ensemble will find those $n_{1}$ and $V_{1}$ for which the chemical potential and pressure of the fluid in the two simulation boxes are equal.

From equation (13) it follows that in a Gibbs-ensemble simulation the pressure of box 1 should be calculated using

$$
\begin{aligned}
P_{1} & \equiv-\left(\frac{\partial A\left(\beta, n_{1}, V_{1}\right)}{\partial V_{1}}\right)_{\beta, n_{1}} \\
& =-\left\langle\frac{\rho_{1}}{\beta}\right\rangle-\left\langle\sum_{i<j=1}^{n_{1}} \frac{1}{3 V} \frac{\mathrm{d} U_{\mathrm{d}}\left(\boldsymbol{r}_{i j}\right)}{\mathrm{d} \boldsymbol{r}_{i j}} \cdot \boldsymbol{r}_{i j}\right\rangle+\left\langle\frac{\rho^{2}}{f(\rho)} \frac{\mathrm{d} f(\rho)}{\mathrm{d} \rho} u_{1}\right\rangle
\end{aligned}
$$

where $u_{1}=\Sigma_{i<j=1}^{n_{1}} U_{\mathrm{d}}\left(\boldsymbol{r}_{i j}\right) / n_{1}$ is the energy per particle. From equation (14) we obtain for the chemical potential of a particle in box 1

$$
\begin{aligned}
\mu_{1} & \equiv\left(\frac{\partial A\left(\beta, n_{1}, V_{1}\right)}{\partial n_{1}}\right)_{\beta, V_{1}} \\
& =-\frac{1}{\beta} \ln \left\langle\frac{V_{1}}{n_{1}+1} \exp \left[-\beta\left(u_{1}^{+}+\frac{\rho}{f(\rho)} \frac{\mathrm{d} f(\rho)}{\mathrm{d} \rho} u_{1}\right)\right]\right\rangle
\end{aligned}
$$

where $u_{1}^{+}$is the energy of a (non-interacting) test particle placed in box 1 . 
These equations differ from the usual expressions for the pressure and chemical potential because the number of particles and the volume appear also in the energy term in the partition function. Appendix B gives a derivation. The equations for the pressure and chemical potential as derived here are not restricted to the Gibbs ensemble; they are also valid for the NVT ensemble.

In this Section we have shown that the Gibbs ensemble can be used for densitydependent potentials and gives the expressions for the chemical potential and pressure. As discussed in the original work of Panagiotopoulos [4], in the Gibbs ensemble it is not necessary to calculate the chemical potential and the pressure. However, equal chemical potential and pressure of the fluid in the two simulation boxes provides an elementary check of the reliability of the simulations. Furthermore, it is important to calculate these quantities if comparisons are made with experimental data.

\section{The Lennard-Jones fluid}

In Section 2, we have shown that the density-dependent potential as defined by equation (2) can be mapped onto a density-independent potential. If the equation of state of the fluid, characterized by the density-independent potential, is known, the equation of state of the fluid, characterized by the density-dependent potential, follows from equation (7). For the Lennard-Jones potential, the equation of state has been determined by Nicolas et al. [8]. The Lennard-Jones fluid provides a suitable system to test the equation derived in this work and to study the influence of density-dependent potentials on the phase diagram.

De Pablo et al. [9] have introduced a density-dependent potential defined by

$$
f\left(\rho^{*}\right)=a \rho^{*}+b
$$

where $\rho^{*}$ is the density in reduced units $\left(\rho^{*}=\rho / \sigma^{3}\right)$ and $a$ and $b$ are constants. In this Section we investigate the influence of a density-dependent potential on the phase diagram of the Lennard-Jones.

\subsection{1. $N, P, T$ simulations}

\subsection{Computer simulations}

We have performed simulations in the $N, P, T$ ensemble for various values of $a$ and $b$. In this ensemble the pressure is specified a priori and the density follows directly from the simulations. Therefore, the results do not depend on equation (15). Table 1 compares the results of these simulations with the predictions from equation (7). Within the accuracy of the simulations, the predicted densities and the results of the simulations are in very good agreement.

\subsubsection{Gibbs ensemble simulations}

We have performed Gibbs-ensemble simulations for $a=0 \cdot 1$ and $b=1$. The results of these simulations are reported in table 2 .

In a simulation with density-independent potentials the pressure is calculated using the virial theorem [1]

$$
p_{\mathrm{vir}}=\left\langle\frac{\rho_{1}}{\beta}\right\rangle-\left\langle\sum_{i<j=1}^{n_{1}} \frac{1}{3 V} \frac{\mathrm{d} U_{\mathrm{d}}\left(\boldsymbol{r}_{i j}\right)}{\mathrm{d} \boldsymbol{r}_{i j}} \cdot \boldsymbol{r}_{i j}\right\rangle .
$$


If one would use equation (18) instead of equation (15), the results for the gas phase would not change. For the coexisting liquid phase, however, one would observe at $T^{*}=1 \cdot 0, P_{\text {vir }}^{*}=0 \cdot 3$; the correct value, as obtained via equation (15), is one order of magnitude smaller! This numerical example emphasizes that the corrections of the conventional $N, V, T$ formula are by no means small. For the chemical potential, the correction is of the order of $15 \%$ at these conditions $\left(T^{*}=1 \cdot 0, P_{\text {vir }}^{*}=0.3\right)$.

Figure 1 shows the effect of the density-dependent potential on the phase diagram. Comparison with the phase diagram for the Lennard-Jones fluid shows that the density dependence has a small effect on the vapour phase. The liquid phase, however, has a considerably higher density. The critical points shifts towards higher temperatures.

In figure 1, we also compare Gibbs-ensemble simulation results with predictions of the equation of state. The equation of state (7) predicts a phase diagram in

Table 1. Comparison of the results from $N, P, T$ simulations with the predictions of the equation of state given by equation (7) coupled with the Nicolas equation of state for the Lennard-Jones fluid. The number of particles in the simulations $N=256$. All simulations were performed at $T^{*}=0.91 \epsilon / k_{\mathrm{B}}$. The pressure and density are given in the usual reduced units: $P^{*}=P \sigma^{3} / \epsilon$ and $\rho^{*}=\rho / \sigma^{3}$. For comparison, some simulations of the Lennard-Jones fluid $(a=0$ and $b=1)$ are added. The subscripts indicate the accuracy of the last digit. $\rho_{\text {eos }}^{*}$ is the reduced density predicted by the equation of state (7) at the indicated pressure.

\begin{tabular}{|c|c|c|}
\hline \multicolumn{2}{|c|}{$N, P, T$ simulations } & \multirow{2}{*}{$\frac{\text { eos }}{\rho_{\text {eos }}^{*}}$} \\
\hline$P^{*}$ & $\rho^{*}$ & \\
\hline \multicolumn{3}{|c|}{$a=0$ and $b=1$} \\
\hline $\begin{array}{l}0.098 \\
0 \cdot 163 \\
0 \cdot 327 \\
0 \cdot 490 \\
0 \cdot 981\end{array}$ & $\begin{array}{l}0 \cdot 759_{9} \\
0 \cdot 764_{11} \\
0 \cdot 779_{7} \\
0 \cdot 796_{11} \\
0 \cdot 826_{8}\end{array}$ & $\begin{array}{l}0.756 \\
0.764 \\
0.780 \\
0.794 \\
0.828\end{array}$ \\
\hline \multicolumn{3}{|c|}{$a=0.082$ and $b=1$} \\
\hline $\begin{array}{l}0 \cdot 098 \\
0 \cdot 163 \\
0 \cdot 327 \\
0 \cdot 490 \\
0 \cdot 981\end{array}$ & $\begin{array}{l}0 \cdot 812_{15} \\
0 \cdot 816_{16} \\
0 \cdot 831_{12} \\
0 \cdot 836_{12} \\
0 \cdot 860_{10}\end{array}$ & $\begin{array}{l}0.812 \\
0.817 \\
0.829 \\
0.839 \\
0.866\end{array}$ \\
\hline \multicolumn{3}{|c|}{$a=-0.082$ and $b=1$} \\
\hline $\begin{array}{l}0.098 \\
0 \cdot 163 \\
0.327 \\
0.490 \\
0.981\end{array}$ & $\begin{array}{l}0.694_{6} \\
0.713_{12} \\
0.737_{9} \\
0.750_{6} \\
0.793_{9}\end{array}$ & $\begin{array}{l}0.690 \\
0.701 \\
0.722 \\
0.742 \\
0.785\end{array}$ \\
\hline
\end{tabular}


Table 2. Results for the Gibbs-ensemble simulations using the density-dependent LennardJones potential with $a=0 \cdot 1$ and $b=1$. The simulations were performed with $N=216$ particles. The total number of Monte Carlo cycles was 15000; the first 5000 were discarded to allow for equilibrium. One Monte Carlo cycle consists of, on average, $N$ attempts to displace a particle in one of the boxes, 150 attempts to exchange a particle, and 1 attempt to change the volume. The algorithm used is a slight modification of the original algorithm of Panagiotopoulos [4,5]; it is described in refs. [10,11]. The result are reported in the conventional reduced units: reduced temperature $T^{*}=T k_{\mathrm{B}} / \epsilon$, reduced density $\rho^{*}=\rho \sigma^{3}$, pressure $P^{*}=P \sigma^{3} / \epsilon$, reduced potential energy per particle $E^{*}=E / \epsilon$, and reduced chemical potential $\mu^{*}=\mu / \epsilon$. Note that the pressure and chemical potential were calculated using equations (15) and (16), respectively. The accuracy of the simulations is indicated via the subscripts.

\begin{tabular}{|c|c|c|c|c|c|c|c|c|}
\hline \multirow[b]{2}{*}{$T^{*}$} & \multicolumn{4}{|c|}{ gas phase } & \multicolumn{4}{|c|}{ liquid phase } \\
\hline & $\rho_{\mathrm{g}}^{*}$ & $P_{\mathrm{g}}^{*}$ & $-\mu_{\mathrm{g}}^{*}$ & $-E_{\mathrm{g}}^{*}$ & $\rho_{1}^{*}$ & $P_{1}^{*}$ & $-\mu_{1}^{*}$ & $-E_{1}^{*}$ \\
\hline 0.909 & $0.009_{3}$ & $0 \cdot 008_{2}$ & $4 \cdot 3_{2}$ & $\mathbf{0} \cdot \mathbf{1}_{1}$ & $0 \cdot 811_{4}$ & $0.02_{2}$ & $4 \cdot 6_{9}$ & $6 \cdot 21_{4}$ \\
\hline 0.950 & $0.010_{3}$ & $0.009_{2}$ & $4 \cdot 4_{1}$ & $0.09_{3}$ & $0.791_{4}$ & $0.02_{2}$ & $4 \cdot 5_{3}$ & $6 \cdot 03_{3}$ \\
\hline $1 \cdot 000$ & $0.019_{4}$ & $0.02_{1}$ & $4 \cdot 2_{1}$ & $0 \cdot 2_{1}$ & $0.77_{1}$ & $0.06_{3}$ & $4 \cdot 5_{4}$ & $5 \cdot 8_{1}$ \\
\hline 1.050 & $0.026_{4}$ & $0.022_{3}$ & $4 \cdot 1_{1}$ & $0.2_{2}$ & $0.750_{5}$ & $0.05_{3}$ & $4 \cdot 1_{1}^{4}$ & $5 \cdot 63_{4}$ \\
\hline $1 \cdot 110$ & $0.035_{9}$ & $0.033_{3}$ & $4 \cdot 0_{1}$ & $0 \cdot 3_{2}$ & $0.727_{6}$ & $0.08_{3}$ & $4 \cdot 1_{2}$ & $5 \cdot 41_{5}$ \\
\hline $1 \cdot 150$ & $0.050_{8}$ & $0 \cdot 05_{1}$ & $3 \cdot 9_{1}$ & $0 \cdot 4_{3}$ & $0 \cdot 707_{7}$ & $0 \cdot 11_{5}$ & $3 \cdot 9_{1}$ & $5 \cdot 22_{3}$ \\
\hline
\end{tabular}

excellent agreement with the simulation results. This agreement makes it unnecessary to perform further simulations using this potential. Results from the equation of state are sufficiently accurate for a variety of practical applications. However, one must be careful for cases in which the Lennard-Jones equation of state is used at temperatures outside the range where this equation has been fitted to simulation data.

\subsection{Density-dependent site-site potentials}

De Pablo et al. [9] introduced the concept of density-dependent potentials to model the thermodynamic properties of various hydrocarbons. As a starting point, they used the site-site Lennard-Jones potentials proposed by Jorgensen et al. [12]. To cover an appreciable range of conditions, de Pablo et al. introduced a density-dependent potential $\epsilon$, defined by

$$
\epsilon / k_{\mathrm{B}}=a \rho+b .
$$

However, the density dependence of the potentials turned out to be unreasonably large. For example, the $\epsilon$ of a carbon site may range from $\epsilon=18 \mathrm{~K}$ in the gas phase to $\epsilon=40 \mathrm{~K}$ in a liquid phase. Since multi-body effects are expected to give rise to only a slight density dependence, these parameters are surprising.

In [9], density-dependent potentials were implemented in a manner different from that used here. Here we assume that, as the density changes in a $N, P, T$ or Gibbsensemble simulation, $\epsilon$ changes according to equation (19) and hence influences the acceptance/rejection of configurations. In the $N, P, T$ simulations of [9] $\epsilon$ was fixed during the production run, but during equilibration $\epsilon$ was manually adjusted using equation (19). A second difference in implementation is that for the Gibbs-ensemble simulations no density-dependent potentials were used. De Pablo et al. assumed that 


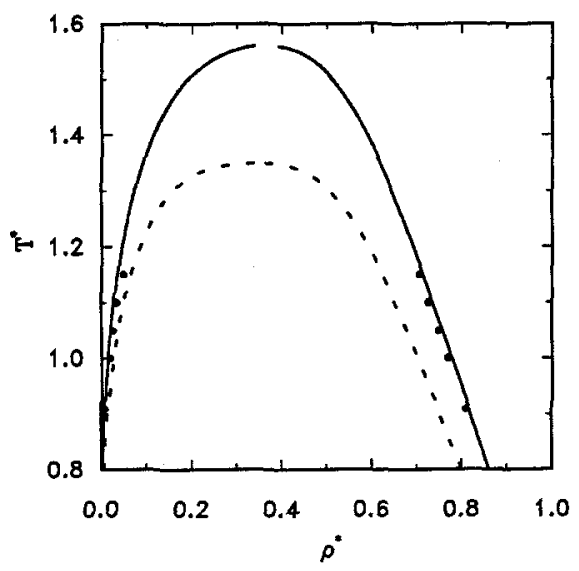

Figure 1. Comparison of the Gibbs-ensemble simulations of the Lennard-Jones fluid with density-dependent potentials $(a=0.1$ and $b=1$, dots $)$ with the prediction of the equation of state (solid line). For comparison, the phase diagram of the Lennard-Jones fluid $a=0$ and $b-1$ ) is also shown (dashed line).

the density dependence has no noticeable effect on the vapour phase and performed Gibbs-ensemble simulations for the coexisting phases using an $\epsilon$ determined only by the liquid-phase density.

Below we demonstrate that fixing $\epsilon$ during the production run is the cause of the unreasonably large density dependence of the parameters reported in [9]. Further, we show that the assumption that Gibbs-ensemble simulations can be performed using the same $\epsilon$ for the liquid and vapour phase results in a phase diagram that is different from the one obtained via a true density-dependent potential.

In [9] site-site potentials were used. However, the procedure of [9] for fitting the parameters is more transparent if we consider an atomic model, e.g. the LennardJones potential. De Pablo et al. used a fixed $\epsilon$ during each production run of their $N, P, T$ simulations. This $\epsilon$ is adjusted iteratively at the beginning of a simulation at a different pressure using equation (19). This way of introducing a sensity-dependent potential is equivalent to a rescaling of the temperature $\left(T^{\text {new }}=T^{*} /\left(a \rho^{*}+b\right)\right.$ at each density but does not correspond to a true density-dependent potential. Assume that we use the Lennard-Jones potential to describe the vapour-liquid equilibrium curve of a fluid. If we fit the Lennard-Jones potential to second-virial-coefficient data, we obtain a fair description of the gas phase, but the density of the liquid phase is generally underestimated significantly. To illustrate, we assume that at $T^{*}=1.00$ the liquid density is underestimated by $11 \%$. We now consider a density-dependent potential to correct the liquid density. If we use the method of [9], the resulting parameters of equation (19) would be $a=0.24$ and $b=1$. If we use, however, a truly density-dependent potential with $a=0.1$ and $b=1$, we arrive at the same liquid density. This example shows why the procedure of de Pablo et al. leads to an unrealistic, large density dependence.

If an Axilrod-Teller formula would be used to estimate the three-body contribution to the energy, it would give a positive contribution to the energy. For the Lennard-Jones potential it turned out to be necessary to introduce a negative contribution to the energy [1]. This suggests that the density-dependent parameters also correct for the deficiencies of the Lennard-Jones potential as indicated by its inability to describe the second virial coefficient of argon within experimental error. 


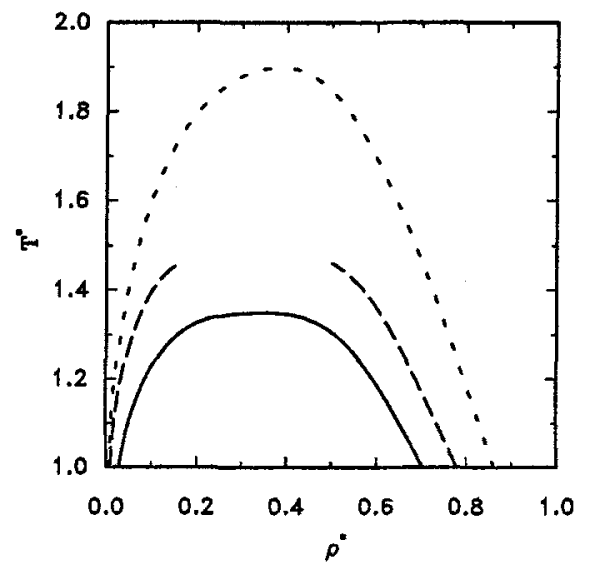

Figure 2. Phase diagram of the Lennard-Jones fluid with density-dependent potential, $a=0.25$ and $b=1.0$ (dotted line). The dashed line is the phase diagram that would have been obtained with Gibbs ensemble simulations using the assumptions of ref. [9]. The solid line gives the phase diagram of the ordinary Lennard-Jones fluid.

We have tested the assumption, used in [9], that Gibbs-ensemble simulations performed with a value of $\epsilon$ that only depends on the density of the liquid phase gives a reasonable approximation to the phase diagram obtained from a density-dependent potential. Figure 2 shows that the two phase diagrams differ appreciably.

\section{Concluding remarks}

In this work we have studied the influence of density-dependent potentials on phase behaviour of simple fluids. It is shown that for a particular class of densitydependent potentials, the equation of state can simply be related to the equation of state of a reference fluid.

This relation is used to study the influence of introducing a density-dependent $\epsilon$ in the Lennard-Jones potential on the phase diagram. We show that a small densitydependence results in a shift of the critical point to a higher temperature and in a higher density of the coexisting liquid phase. The influence on the vapour phase is small.

Computer simulations in the Gibbs or $N, V, T$ ensemble using density-dependent potentials require slightly different expressions for the pressure and chemical potential. Numerical examples show that significant errors are made if these correction on the conventional expressions are ignored.

This work is restricted to pure components. For mixtures or for molecular fluids, the mapping of the potential onto a density-independent potential can not be used.

We have shown that the procedure for implementing density-dependent potentials given here present a significant improvement over that recently proposed in [9]. While the results presented there show excellent agreement with experimental data, our study indicates that this agreement could only be achieved through introduction

$\uparrow$ This potential is density-independent in the sense that the same value of $\epsilon$ is taken for the liquid and vapour phase at a given temperature. The value of the $\epsilon$ at a given temperature is calculated from equation (19) using the $\rho$ the density of the coexisting liquid phase. 
of an unrealistic large density dependence to compensate for inconsistencies in the implementation of density-dependent potentials.

The authors thank J. J. de Pablo, A. Z. Panagiotopoulos, D. Frenkel, H. C. Anderson, and D. Chandler for their contributions to this work. Torsten Hauschild wishes to express his gratitude to the Alexander-von-Humboldt Foundation, for financial support. This project was supported by the Director, Office of Energy Research, Office of Basic Energy Sciences, Chemical Sciences Division of the US Department of Energy under contract No. DE-ACO3-76SF00098.

\section{Appendix A: the equation of state}

This Appendix gives a detailed derivation of the equation of state for a fluid described with a density-dependent potential $U_{\mathrm{d}}$ defined by equation (2). In Section 2 , we have shown that equation (5) gives the relation between the partition functions of the fluid described with a density-dependent potential and a fluid described with a density-independent potential $U_{0}$. The Helmholtz energy $A$ is related to the partition function via

$$
A=-\frac{1}{\beta} \ln Z
$$

Using equations (5), (6), and (20), we can derive for the pressure

$$
\begin{aligned}
P(\beta, \rho) \equiv & -\left(\frac{\partial A}{\partial V}\right)_{\beta, N}=\frac{1}{\beta} \frac{1}{Z}\left(\frac{\partial Z(\beta, N, V)}{\partial V}\right)_{\beta, N} \\
= & \frac{1}{\beta f(\rho)^{(3 / 2) N}} \frac{1}{Z_{0}}\left(\frac{\partial f(\rho)^{(3 / 2) N} Z_{0}\left(\beta^{\prime}, N, V\right)}{\partial V}\right)_{\beta, N} \\
= & \frac{1}{\beta f(\rho)^{(3 / 2) N}} \frac{1}{Z_{0}}\left\{Z_{0}\left(\frac{\partial f(N / V)^{(3 / 2) N}}{\partial V}\right)_{\beta, N}\right. \\
& \left.\left.+f(\rho)^{(3 / 2) N}\left(\frac{\partial z_{0}\left(\beta^{\prime}, N, V\right)}{\partial v}\right)_{\beta, N}\right)_{V, N}\left(\frac{\partial f(N / V)}{\partial V}\right)_{\beta, N}\right\} \\
& +\beta f(\rho)^{(3 / 2) N}\left(\frac{\partial z_{0}\left(\beta^{\prime}, N, V\right)}{\partial \beta^{\prime}}\right\} \\
= & -\frac{3}{2} \frac{\rho^{2}}{\beta f(\rho)} \frac{\mathrm{d} f(\rho)}{\mathrm{d} \rho}+f(\rho) P_{0}\left(\beta^{\prime}, \rho\right)+\rho^{2} \frac{\mathrm{d} f(\rho)}{\mathrm{d} \rho} u_{0}\left(\beta^{\prime}, \rho\right),
\end{aligned}
$$

where $u_{0}=U_{0} / N$ is the energy per particle of the reference system which is related to partition function $Z_{0}$ according to

$$
U_{0}=\frac{1}{Z_{0}} \int \mathrm{d} \Gamma U_{0} \exp \left(-\beta U_{0}\right)=-\frac{1}{Z_{0}}\left(\frac{\partial Z_{0}}{\partial \beta}\right)_{N, V}
$$

Equation (A 2) shows that the equation of state of the fluid with a density-dependent potential can be expressed in terms of the equation of state and energy of the reference fluid. 


\section{Appendix B: The pressure and chemical potential}

Here we derive the expressions for the chemical potential and pressure for a simulation in the Gibbs ensemble. In Section 3, we have shown that the pressure in one of the boxes of the Gibbs ensemble is defined by

$$
P_{1} \equiv-\left(\frac{\partial A\left(\beta, n_{1}, V_{1}\right)}{\partial V_{1}}\right)_{\beta, n_{1}}
$$

and the chemical potential by

with

$$
\mu_{1} \equiv\left(\frac{\partial A\left(\beta, n_{1}, V_{1}\right)}{\partial n_{1}}\right)_{\beta, V_{1}}
$$

$$
\exp (-\beta A)=Z\left(\beta, n_{1}, V_{1}\right)=\frac{1}{\Lambda^{3 n_{1}} n_{1} !} \int \mathrm{d} \Gamma_{1}^{n_{1}} \exp \left[-\beta f\left(n_{1} / V_{1}\right) U_{0}\right]
$$

From these expressions we can derive the ensemble averages that can be calculated in a simulation.

For the pressure we can write

$$
P_{1}=-\left(\frac{\partial A\left(\beta, n_{1}, V_{1}\right)}{\partial V_{1}}\right)_{\beta, n_{1}}=\frac{1}{3 \beta Z L_{1}^{2}}\left(\frac{\partial Z\left(\beta, n_{1}, L_{1}^{3}\right)}{\partial L_{1}}\right)_{\beta, n_{1}},
$$

where $L_{1}^{3}=V_{1}$. If we introduce scaled coordinates $\xi_{i}=r_{i} / L_{1}$, we can write for the partition function

$$
Z\left(\beta, n_{1}, L_{1}\right)=\frac{L_{1}^{3 n_{1}}}{\Lambda^{3 n_{1}} n_{1} !} \int \mathrm{d} \xi^{n_{1}} \exp \left[-\beta f\left(n_{1} / L_{1}^{3}\right) U_{0}\left(\xi L_{1}\right)\right] .
$$

This gives for the pressure

$$
\begin{aligned}
P_{1} & =\frac{3 n_{1} Z}{3 L_{1}^{2} L_{1} \beta Z}+\frac{1}{3 \beta L_{1}^{2}}\left\langle\frac{\partial \exp \left[-\beta f\left(n_{1} / L_{1}^{3}\right) U_{0}\left(\xi L_{1}\right)\right]}{\partial L_{1}}\right\rangle \\
& =\left\langle\frac{\rho_{1}}{\beta}\right\rangle-\left\langle\sum_{i<j=1}^{n_{1}} \frac{1}{3 V_{1}} \frac{\mathrm{d} U_{\mathrm{d}}\left(r_{i j}\right)}{\mathrm{d} r_{i j}} \cdot r_{i j}\right\rangle+\left\langle\frac{\rho_{1}^{2}}{f\left(\rho_{1}\right)} \frac{\mathrm{d} f\left(\rho_{1}\right)}{\mathrm{d} \rho_{1}} u_{1}\right\rangle,
\end{aligned}
$$

where $u_{1}=\Sigma_{i<j=1}^{n_{1}} U_{\mathrm{d}}\left(\boldsymbol{r}_{i j}\right) / n_{1}$ is the energy per particle.

For the chemical potential we have

$$
\begin{gathered}
\beta \mu_{1}=\beta\left[A\left(\beta, n_{1}+1, V_{1}\right)-A\left(\beta, n_{1}, V_{1}\right)\right]=-\ln \frac{Z\left(\beta, n_{1}+1, V_{1}\right)}{Z\left(\beta, n_{1}, V_{1}\right)} \\
=-\ln \left\{\frac{1}{\left(n_{1}+1\right) \Lambda^{3}} \frac{\int \mathrm{d} \Gamma_{1}^{n_{1}+1} \exp \left[-\beta f\left(\left(n_{1}+1\right) / V_{1}\right) U_{0}\right]}{\int \mathrm{d} \Gamma_{1}^{n_{1}} \exp \left[-\beta f\left(n_{1} / V_{1}\right) U_{0}\right]}\right\} \\
=-\ln \left(\frac{V_{1}}{\left(n_{1}+1\right) \Lambda^{3}}\right. \\
\left.\times \frac{\int \mathrm{d} \Gamma_{1}^{n_{1}} \exp \left[-\beta\left\{u_{1}^{+}+\left[f\left(\left(n_{1}+1\right) / V_{1}\right)-f\left(n_{1} / V_{1}\right)\right] U_{0}\right\}\right] \exp \left[-\beta f\left(n_{1} / V_{1}\right) U_{0}\right]}{\int \mathrm{d} \gamma_{1}^{n_{1}} \exp \left[-\beta f\left(n_{1} / V_{1}\right) U_{0}\right]}\right) \\
=-\ln \left\langle\frac{V_{1}}{n_{1}+1} \exp \left\{-\beta\left(u_{1}^{+}+\frac{\rho_{1}}{f\left(\rho_{1}\right)} \frac{\mathrm{d} f\left(\rho_{1}\right)}{\mathrm{d} \rho_{1}} u_{1}\right)\right\}\right\rangle-3 \ln \Lambda,
\end{gathered}
$$


where $u_{1}=U_{1} / n_{1}$ is the energy per particle and $u_{1}^{+}$is the energy of a (non-interacting) test particle that is added to box 1.

\section{References}

[1] Allen, M. P., and Tildesley, D. J., 1987, Computer Simulation of Liquids (Oxford: Clarendon).

[2] Barker, J. A., Fisher, R. A., and Watts, R. O., 1971, Molec. Phys., 21, 657.

[3] Monson, P. A., Rigby, M., and Steele, W. A., 1983, Molec. Phys., 49, 893.

[4] Panagiotopoulos, A. Z., 1987, Molec. Phys., 61, 813.

[5] Panagiotopoulos, A. Z., Quirke, N., Stapleton, M., and Tildesley, D. J., 1988, Molec. Phys., 63, 527.

[6] Smit, B., De Smedt, Ph., and Frenkel, D., 1989, Molec. Phys., 68, 931.

[7] Panogiotopoulos, A. Z., 1992, Molec. Simulation, 9, 1.

[8] Nicolas, J. J., Gubbins, K. E., Streett, W. B., and Tildesley, D. J., 1979, Molec. Phys., 37, 1429.

[9] de Pablo, J. J., Bonnin, M., and Prausnitz, J. M., 1992, Fluid Phase Equilibria, 73, 187.

[10] Sмiт, B., 1990, Computer Simulation of Phase Coexistence: from Atoms to Surfactants, $\mathrm{PhD}$ thesis, Rijksuniversiteit Utrecht, The Netherlands.

[11] Smit, B., and Frenkel, D., 1991, J. chem. Phys., 94, 5663,

[12] Jorgensen, W. L., Madura, J. D., and Swenson, C. J., 1984, J. Am. Chem. Soc., 106, 6638. 